\title{
Noise Attenuation from GPR Data Using Wavelet Transform and Artificial Neural Network
}

\author{
Sid-Ali Ouadfeul ${ }^{1 *}$, Leila Aliouane ${ }^{2}$ \\ ${ }^{1}$ Algerian Petroleum Institute, IAP, Avenue 1er Novembre. Boumerdes, 35000, Algerian. \\ 2 Leila Aliouane, Labophyt, FHC, UMBB, Avenue de l'indépenance, 35000, Boumerdes, Algeria. \\ * Corresponding author. Tel.: +213560216625; email: SOuadfeul@ymail.com \\ Manuscript submitted July 28, 2014; accepted November 23, 2014. \\ doi: 10.17706/ijapm.2014.4.6.426-433
}

\begin{abstract}
In this paper, a tentative of noise attenuation from the 3D Ground Penetrating Radar (GPR) data using the wavelet transform and the Multilayer Perceptron neural network model is implanted. GPR data recorded in Algerian Sahara are filtered firstly using the discrete wavelet transform, after that a MLP machine with three layers is trained in a supervised learning mode, the input is an extracted profile from the raw 3D GPR data and the output consists of the GPR data of the same profile but after filtering. The estimated weights of connection are used to propagate the remaining non-filtered data through the implanted machine, the calculated output consists to the filtered GPR data. Comparison between the filtered data using the MLP machine and the continuous wavelet transform shows that the neural network machine can be used for S/N ratio improvement of the noisy GPR data.
\end{abstract}

Key words: Noise, attenuation, GPR, discrete wavelet transform, neural network, multilayer perceptron.

\section{Introduction}

Wavelet transform has becoming an important and useful signal processing tool in geophysics, it has been used for causative sources characterization from potential gravity and magnetic field [1]. In petrophysics, the wavelet transform was used for lithofacies segmentation [2], In ground penetrating radar field, it has been used for helping 3D seismic design by mapping maxima of the modulus of the continuous wavelet transform for all scales [3].

Artificial Neural Networks have been widely used in geophysical data processing, it has been used for lithofacies classification form well-logs data [4], and in the prediction of petrophysical parameters like porosity, permeability and water saturation [5].

Ground Penetrating Radar (GPR) data filtering has becoming a very interesting subject of research. Kim et al. [6] have suggested removing the ringing noise in GPR data by signal processing; they have shown that that ringing can be successfully removed by the eigenimage. Lopera et al. [7] have proposed a novel approach to filter out these effects from 2-D off-ground monostatic GPR data by adapting and combining the radar antenna subsurface model of Lambot with phase-shift migration. Jeng et al. [8] have suggested an adaptive filtering technique of random noise in near-surface seismic and ground-penetrating radar data. In this paper, we propose another method to filter GPR data from random noise, it is based on the combination between the discrete wavelet transform and the Multilayer Perceptron (MLP) neural network model.

We start the paper by describing the principle of the Discrete Wavelet Transform and how it can be used 
for signal denoising. After that we describe the principle of the Maultilayer Perceptron neuran network, and the processing algorithm. The proposed idea is then applied to real GPR data recorded in the Algerian Sahara. We end the paper by results interpretation and a conclusion.

\section{The Continuous Wavelet Transform}

Here we review some of the important properties of wavelets, without any attempt at being complete. What makes this transform special is that the set of basis functions, known as wavelets, are chosen to be well-localized (have compact support) both in space and frequency [9]. Thus, one has some kind of "dual-localization" of the wavelets. This contrasts the situation met for the Fourier's transform where one only has "mono-localization", meaning that localization in both position and frequency simultaneously is not possible.

The CWT of a function s(z) is given by Grossmann and Morlet, [4] as:

$$
C_{S}(a, b)=\frac{1}{\sqrt{a}} \int_{-\infty}^{+\infty} s(z) \psi^{*}(z) d z
$$

Each family test function is derived from a single function $\psi(z)$ defined to as the analyzing wavelet according to [9]:

$$
\psi_{a, b}(z)=\psi\left(\frac{z-b}{a}\right)
$$

where $a \in R^{+*}$ is a scale parameter, $b \in R$ is the translation and $\psi^{*}$ is the complex conjugate of $\psi$. The analyzing function $\psi(z)$ is generally chosen to be well localized in space (or time) and wavenumber. Usually, $\psi(z)$ is only required to be of zero mean, but for the particular purpose of multiscale analysis $\psi(z)$ is also required to be orthogonal to some low order polynomials, up to the degree $n-1$, i.e., to have $n$ vanishing moments:

$$
\int_{-\infty}^{+\infty} z^{n} \psi(z) d z=0 \text { for } 0 \leq n \leq p-1
$$

According to this equation, $p$ order moment of the wavelet coefficients at scale a reproduce the scaling properties of the processes. Thus, while filtering out the trends, the wavelet transform reveals the local characteristics of a signal, and more precisely its singularities.

\section{Discrete Wavelet Transform and Signal Denoising}

The function $\psi(t)$ is said to be a wavelet if and only when the following condition is satisfied:

$$
\int_{-\infty}^{+\infty} \psi(t) d t=0
$$

The wavelet transform of a function $\psi(t) \in L^{2}\left(R^{2}\right)$ is defined by [4]:

$$
\psi_{a} f(t)=f(t) * \psi_{a}(t)
$$


where $\quad \psi_{a}(t)=\frac{1}{a} \psi_{a}\left(\frac{t}{a}\right) \quad$ is the dilated version of $\psi(t)$ by the scale factor $a$.

In practice $a$ is scattered as discrete binary system, i.e. Let $a=2^{j}(j \in Z)$, then the wavelet is $\psi_{2^{j}}(t)=\frac{1}{2^{j}} \psi_{2^{j}}\left(\frac{t}{2^{j}}\right) \quad$,its wavelet transform is given by:

$$
W_{2}{ }^{j} f(t)=f(t) * \psi_{2} j(t)=f(t) * \frac{1}{2^{j}} \psi\left(\frac{t}{2^{j}}\right)
$$

Hence its contrary transform is $f(t)=\sum_{-\infty}^{+\infty} W_{2^{j}} f(t) * x(t)$. Where $x(t)$ satisfies $\sum_{-\infty}^{+\infty} \hat{\psi}\left(2^{j} w\right) x\left(2^{j} w\right)=1$.

Being dispersed in time domain farther, a discrete wavelet transform can be obtained. It exists an effective and fast algorithm based on equation (7)

$$
\begin{gathered}
S_{2^{j}} f=S_{2^{j-1}} f^{*} H_{j-1} \\
W_{2} f=S_{2}{ }^{j-1} f^{*} G_{j-1}
\end{gathered}
$$

There $W_{2}^{j} f$ is the wavelet transform coefficients of $f(t)$.

It approximates $f(t)$ on the scale $2^{\mathrm{j}}$

$H_{j}$ and $G_{j}$ are the discrete filters gained by inserting $\left(2^{j-} 1\right)$ zeros into every two samples of $\mathrm{H}, \mathrm{G}$. The relation between $G$ and $H$ is:

$$
g_{k}=(-1)^{k-1} \bar{h}_{1-k}
$$

Thresholding is a technique used for signal and image denoising [4]. The discrete wavelet transform uses two types of filters: (1) averaging filters, and (2) detail filters. When we decompose a signal using the wavelet transform, we are left with a set of wavelet coefficients that correlates to the high frequency sub-bands. These high frequency sub-bands consist of the details in the data set. If these details are small enough, they might be omitted without substantially affecting the main features of the data set. Additionally, these small details are often those associated with noise; therefore, by setting these coefficients to zero, we are essentially killing the noise. This becomes the basic concept behind thresholding-set all frequency sub-band coefficients that are less than a particular threshold to zero and use these coefficients in an inverse wavelet transformation to reconstruct the data set.

\section{The Multilayer Perceptron}

Multilayer feed-forward networks form an important class of neural networks. Typically the network consists of a set of sensory units or input nodes, that constitute the input layer, one or more hidden layers of neurons or computation nodes, and an output layer. Multi-layer Perceptron (MLP) neural networks with sufficiently many nonlinear units in a single hidden unit layer have been established as universal function approximators. The advantages of the MLP are: Hidden unit outputs (basis functions) change adaptively during training, making it unnecessary for the user to choose them beforehand. The number of free parameters in the MLP can be unambiguously increased in small increments by simply increasing the number of hidden units. The basic functions are bounded making overflow errors and round-off errors unlikely. The MLP is a feed-forward network consisting of units arranged in layers with only forward connections to units in subsequent layers. The connections have weights associated with them. Each signal 
traveling along a link is multiplied by its weight. The input layer, being the first layer, has input units that distribute the inputs to units in subsequent layers. In the following (hidden) layer, each unit sums its inputs and adds a threshold to it and nonlinearly transforms the sum (called the net function) to produce the unit output (called the activation). The output layer units often have linear activations, so that output activations equal net function values. The layers sandwiched between the input and the output layers are called hidden layers, and the units in the hidden layers are called hidden units [5].

\subsection{Modified Hidden Weight Optimization Algorithm}

The output weight optimization-hidden weight optimization (OWO-HWO) feed forward network training algorithm alternately solves linear equations for output weights and reduces a separate hidden layer error function with respect to hidden layer weights. Here, a new hidden layer error function is proposed which de-emphasizes net function errors that correspond to saturated activation function values. In addition, an adaptive learning rate based on the local shape of the error surface is used in hidden layer training. For more details about the HWO algorithm, we invite authors to the paper of Yu and Manry [10].

\section{Ground Penetrating Radar}

Ground-penetrating radar (GPR) is a geophysical method that uses radar pulses to image the subsurface. This nondestructive method uses electromagnetic radiation in the microwave band (UHF/VHF frequencies) of the radio spectrum, and detects the reflected signals from subsurface structures. GPR can be used in a variety of media, including rock, soil, ice, fresh water, pavements and structures. It can detect objects, changes in material, and voids and cracks. GPR uses high-frequency (usually polarized) radio waves and transmits into the ground. When the wave hits a buried object or a boundary with different dielectric constants the receiving antenna records variations in the reflected return signal. The principles involved are similar to reflection seismology, except that electromagnetic energy is used instead of acoustic energy, and reflections appear at boundaries with different dielectric constants instead of acoustic impedances.

The depth range of GPR is limited by the electrical conductivity of the ground, the transmitted center frequency and the radiated power. As conductivity increases, the penetration depth decreases. This is because the electromagnetic energy is more quickly dissipated into heat, causing a loss in signal strength at depth. Higher frequencies do not penetrate as far as lower frequencies, but give better resolution. Optimal depth penetration is achieved in ice where the depth of penetration can achieve several hundred meters. Good penetration is also achieved in dry sandy soils or massive dry materials such as granite, limestone, and concrete where the depth of penetration could be up to 15 -metre ( $49 \mathrm{ft}$ ). In moist and/or clay-laden soils and soils with high electrical conductivity, penetration is sometimes only a few centimeters.

Ground-penetrating radar antennas are generally in contact with the ground for the strongest signal strength; however, GPR air-launched antennas can be used above the ground.

Cross borehole GPR has developed within the field of hydro geophysics to be a valuable means of assessing the presence and amount of soil water.

\section{The Processing Algorithm}

The processing algorithm is based on the application of the discrete wavelet transform to filter GPR data form random noise, after that a multilayer perceptron (MLP) neural network machine is trained in a supervised mode. The input is the noisy GPR data. However the output is the filtered GPR data using the DCWT. The training stage consists to weights of connection determination. The implanted neural network machine will be used to filter the remaining GPR data, without needs to calculate the weights of connection, the noisy GPR data will be propagated through the neural network to calculate an output. This output presents the filtered GPR data. 


\section{Application to Real Data}

The proposed idea is applied to 3D GPR data recorded in Algeria, these data presents the altimetry of the area versus $\mathrm{X}$ and $\mathrm{Y}$ (see Fig. 1). The image acquisition is done with a central frequency of $50 \mathrm{MHZ}$. The data are recorded in 49 profiles, the spatial spacing between two profiles is $250 \mathrm{~m}$, and the distance between two points in the same profile is $50 \mathrm{~m}$. Figure $01 \mathrm{~b}$ shows the noisy GPR data with $05 \%$ of white noise. Fig. 2 shows first GPR profile extracted from the GPR image. The discrete wavelet transform combined with the thresholding technique are used to denoise this GPR profile, the obtained result is compared with the original GPR profile without noise in figure 03. It is clear that the discrete wavelet transform is able to denoise the GPR data, however amplitudes are not preserved. The next step in the processing algorithm consists to train a multilayer perceptron neural network machine in a supervised learning mode, the noisy GPR profile is sub-dived in 33 sets, and each set is composed of ten elements. These sets of data are used as an input to train the MLP machine. The denoised GPR data are sub-divided in the same way and used as an output for supervised training of the MLP machine. The training of neural machine composed of three layers, an input layer with ten neurons, an output layer is ten neurons and a hidden layer with nine neurons is done in 33 iteration, For each iteration the Root Mean Square (RMS) error is calculated and the training algorithm, the training algorithm is the so-called OWO-HWO [5].

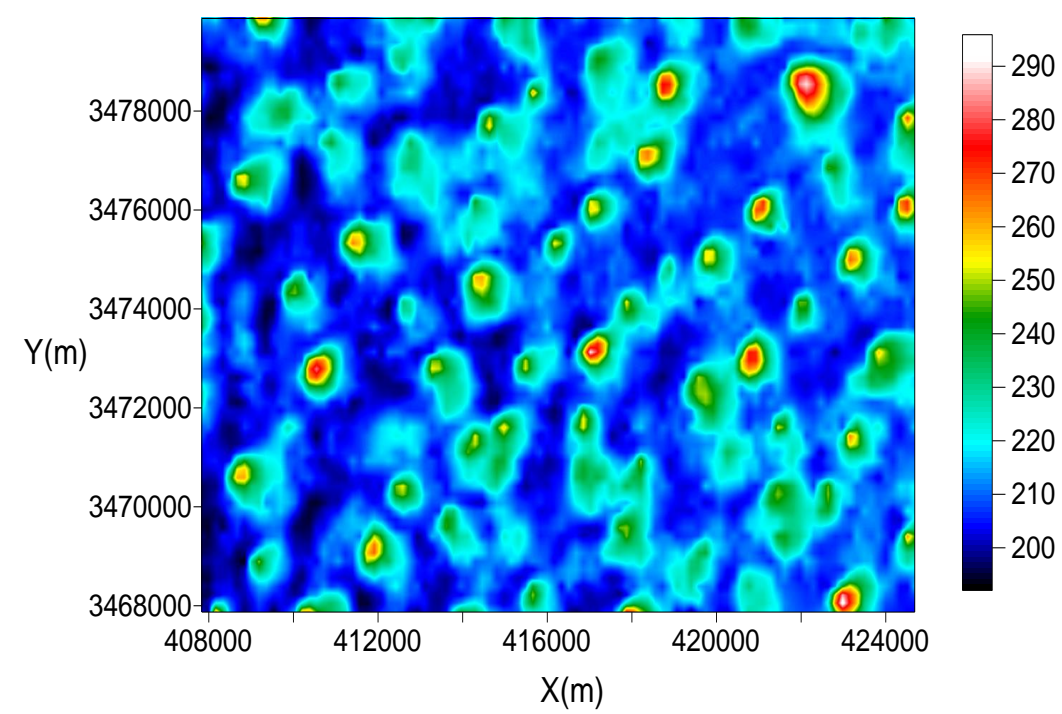

a)

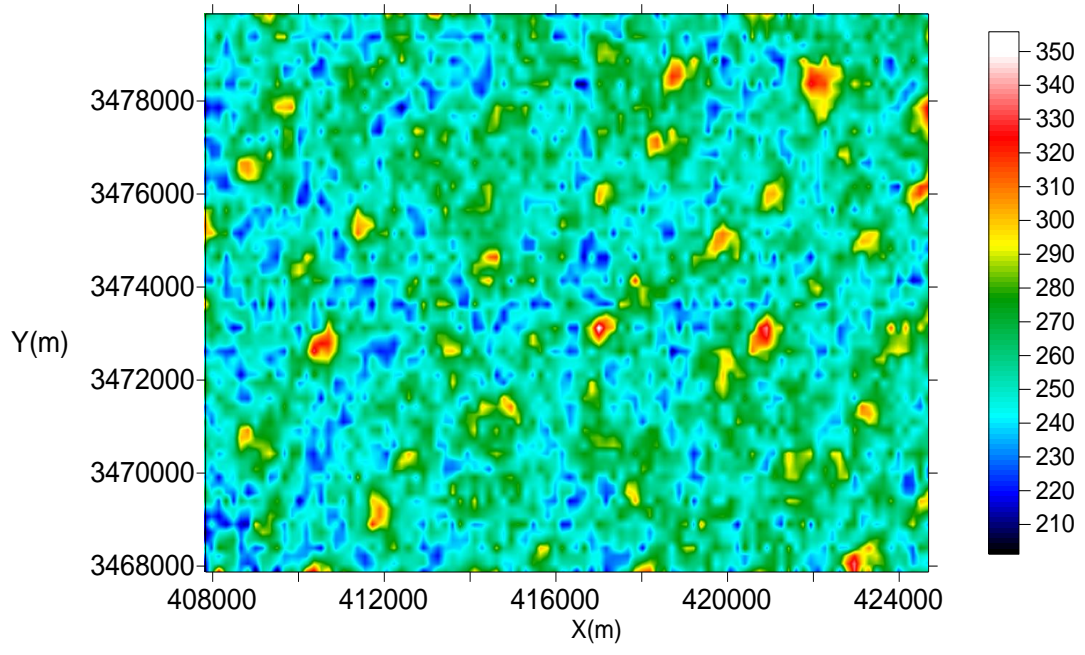

b)

Fig. 1 a) Initial 3D GPR image without noise. b) Noisy 3D GPR Image. 


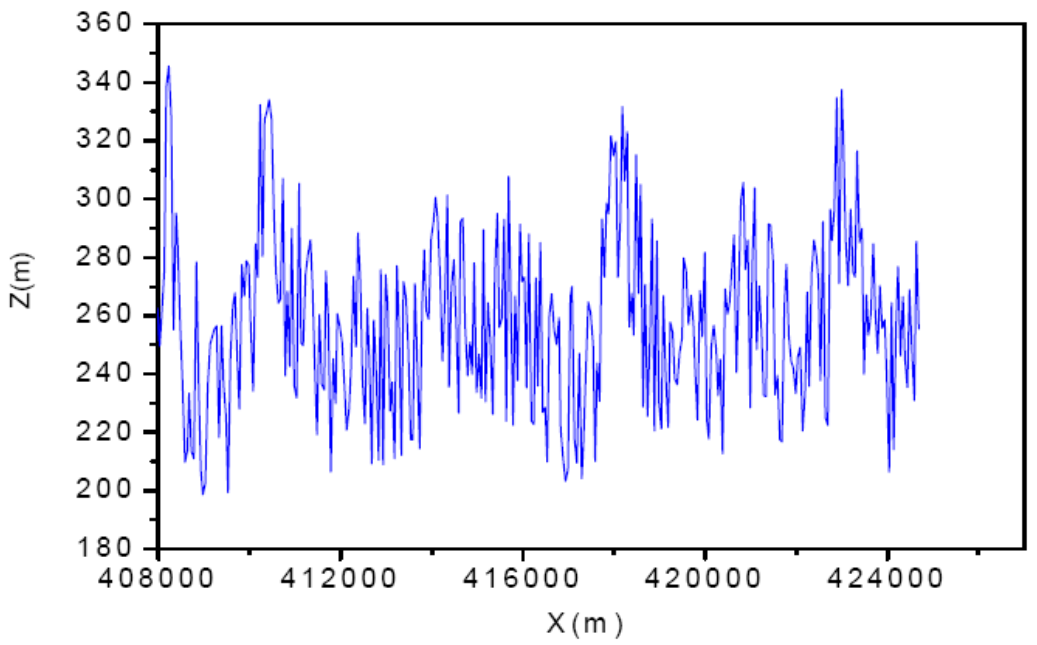

Fig. 2 First GPR profile extracted from the 3D GPR image.

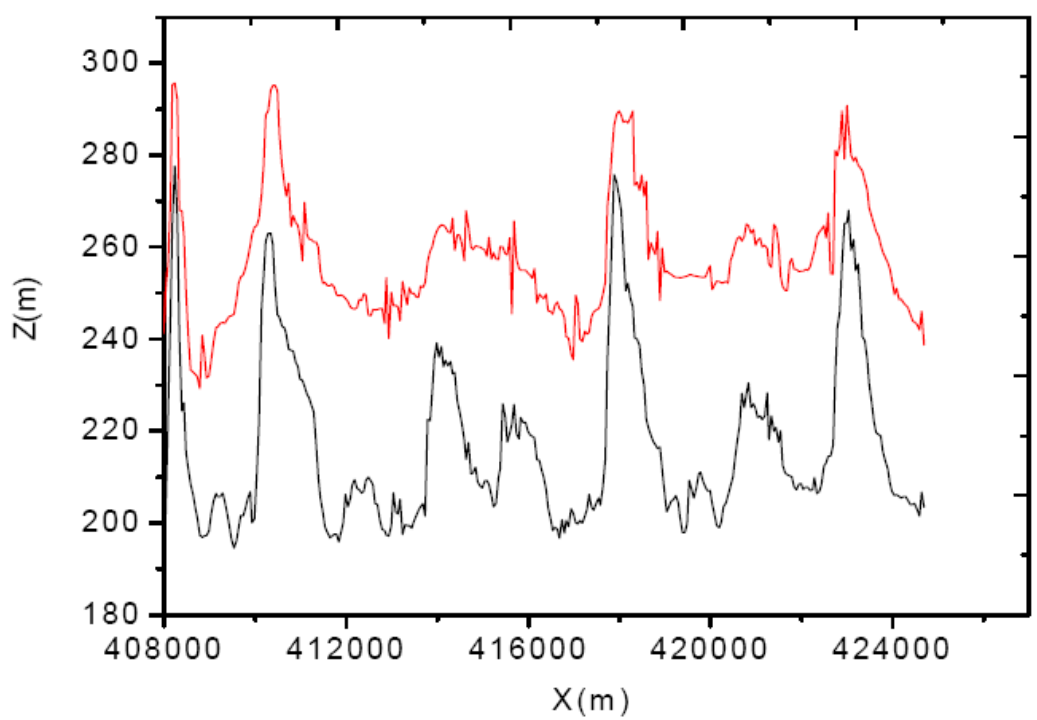

Fig. 3. Denoised GPR profile (red color) compared with the original profile (black color).

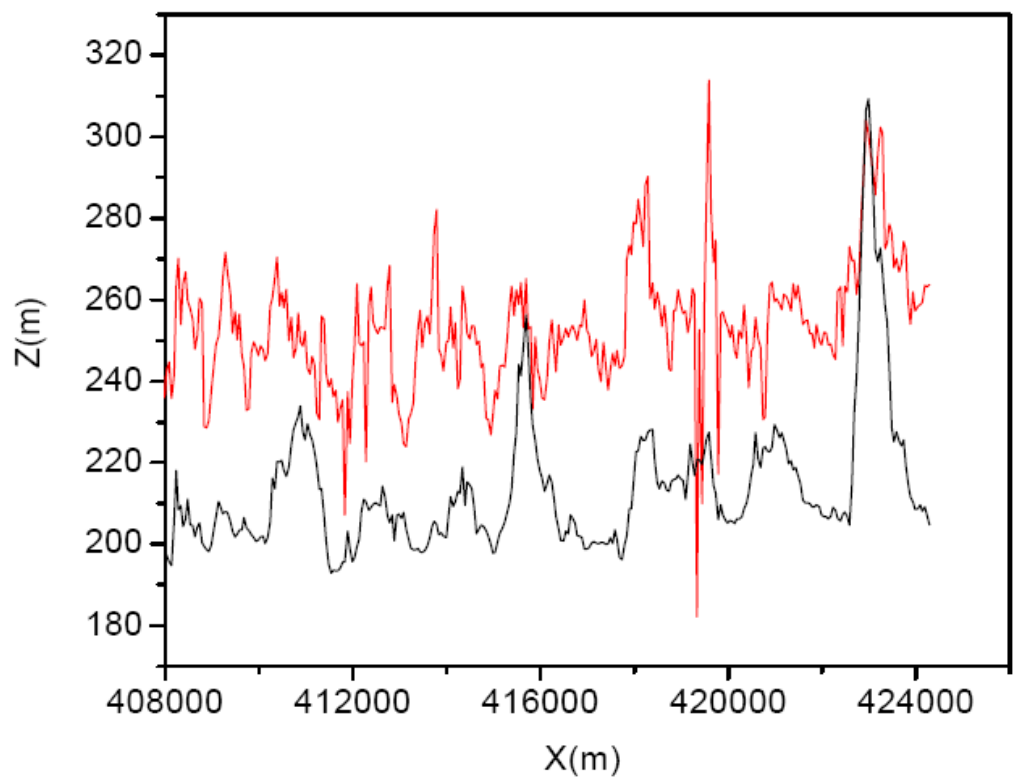

Fig. 4. Denoised second GPR profile using the multilayer perceptron compared with the original profile. 


\section{Results and Interpretation}

During the supervised training of the multilayer perceptron neural network machine the weights of connections are calculated, the GPR data used as input are propagated through the neural machine and an output is calculated. Fig. 3 shows a comparison between the calculated and the actual outputs of the neural machine.

It is clear that the MLP machine is able to provide good GPR data with good S/N ratio which proves the good learning quality of the MLP machine. The weights of connections are used to denoise the second GPR profile extracted from figure 01 , at this stage the noisy GPR data are propagated through the neural network machine and no desired output is needed. Obtained results are compared with the same GPR profile but without noise (see figure 04). This figure shows clearly that the MLP machine is able denoise the GPR data however the whole process is not able to preserve amplitudes. The obtained results show that we have implanted a neural network machine that can be used to improve the $\mathrm{S} / \mathrm{N}$ ratio of the whole 3D GPR data presented in figure 01 . We recommend testing other neural network models like the radial basis function and the feed-forward to compare and improve the GPR data filtering methods and techniques.

\section{Conclusion}

We have implanted a neural network machine able to filter the ground penetrating radar data from the random noise, the neural machine is a multilayer perceptron kind. It is composed with three layers an input layer with ten neurons and an output layer with ten neurons. The training algorithm is the so-called the OWO-HWO. The implanted machine is applied to 3D GPR data recorded in Algeria, obtained results clearly shows the robustness of the implanted machine to attenuate the random noise from the GPR data.

Data propagation of another GPR profile clearly shows the ability of the MLP machine to filter the data from the random noise. However the machine is not able to preserve amplitudes, to resolve this ambiguity we suggest application of again to amplitudes, the goal is to preserve amplitudes during the processing.

We recommend application of other neural network models such the radial basis function to compare between neural network machines and to choose the best machine that is able to provide better results.

\section{References}

[1] Ouadfeul, S., Aliouane, L., Hamoudi, M., Boudella, A., \& Eladj, S., (2012). 1d wavelet transform and geosciences. In Dumitru Baleanu (Eds.), Wavelet Transforms and Their Recent Applications in Biology and Geoscience.

[2] Ouadfeul, S., \& Aliouane, L. (2011). Multifractal analysis revisited by the continuous wavelet transform applied in lithofacies segmentation from well-logs data. International Journal of Applied Physics and Mathematics, 1(1).

[3] Ouadfeul, S. (2010). Multiscale analysis of 3D GPR data using the continuous wavelet transform. IEE Xplore Compliance.

[4] Ouadfeul, S., \& Aliouane, L. (2012). Lithofacies classification using the multilayer perceptron and the self-organizing neural networks. Lecture Notes in Computer Science, 7667, 737-744.

[5] Aliouane, L., Ouadfeul, S., Djarfour, N., \& Boudella, A. (2012). Petrophysical parameters estimation from well-logs data using multilayer perceptron and radial basis function neural networks. Lecture Notes in Computer Science, 7667, 730-736.

[6] Kim, J.-H., Cho, S.-J., \& Yi, M.-J. (2007). Removal of ringing noise in GPR data by signal processing. Geosciences Journal, 11(1), 75-81.

[7] Lopera, O., Slob, E.-C., Milisavljevic, M., \& Lambot, S. (2007). Filtering soil surface and antenna effects from GPR data to enhance landmine detection. IEEE Transactions on Geoscience and Remote Sensing, 45(3). 
[8] Jeng, Y., Li, Y.-W., Chen, C.-S., \& Chien. H-Y. (2009). Adaptive filtering of random noise in near-surface seismic and ground-penetrating radar data. Journal of Applied Geophysics, 68, 36-46.

[9] Ouadfeul, S., \& Aliouane, L. (2013). Random seismic noise attenuation data using the discrete and the continuous wavelet transforms. Arab Journal of Geosciences.

[10] Yu, C., \& Manry, M. T. (2002). A modified hidden weight optimization algorithm for feedforward neural networks. Proceedings of Conference Record of the Thirty-Sixth Asilomar Conference on Signals, Systems and Computers.

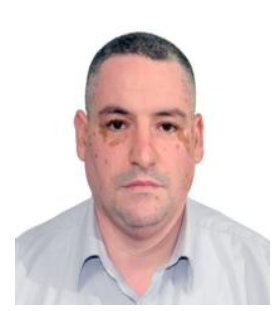

Sid-Ali Ouadfeul is currently a professor at the Department of Geophysics, Geology and Reservoir Engineering, Algerian Petroleum Institute-IAP Corporate University, Algeria. He received his engineer diploma from University of Boumerdes, Algeria, a magister and the PhD in geophysics from the Faculty of Earth Sciences, USTHB, Algeria. During his master and doctorate research he has developed many techniques based on the fractal analysis to understand the earth underlying system. His research interests focus mainly on the studies of nonlinear process in geosciences including the fractal analysis, wavelet transform and artificial intelligence. He is an author of many papers on the subject. Dr. Ouadfeul is the organizer of many sessions in international conferences over the world about the understanding of chaotic systems in Geophysics. He serves as an editorial member of many reputable journals.

Leila Aliouane is currently a researcher at the University of Boumerdes. Her main research field is the pattern recognition in geophysics using petro physical and seismic data. She has organized many conferences on the topic. She is an author of many papers on the artificial neural network and pattern recognition. 